\title{
Digital ureteroscopes: technology update
}

This article was published in the following Dove Press journal:

Research and Reports in Urology

27 January 2017

Number of times this article has been viewed

\section{Chad M Gridley \\ Bodo E Knudsen}

Department of Urology, The Ohio State University Wexner Medical Center, Columbus, OH, USA
Correspondence: Bodo E Knudsen Department of Urology, The Ohio State University Wexner Medical Center, 915 Olentangy River Rd, Columbus, $\mathrm{OH}$ 43212, USA

Tel +l 6142939349

Fax + I 6I4 2933565

Email Bodo.Knudsen@osumc.edu
Abstract: The field of ureteroscopy has undergone a continual evolution since the first ureteroscopes were introduced. Over the past 10 years, we have entered into the digital era of ureteroscopy with both semirigid and flexible options becoming available. The following review looks at the benefits and drawbacks of digital flexible ureteroscopes as well as the current commercially available options.

Keywords: digital, video, flexible ureteroscopy

\section{Introduction}

The field of urology has seen numerous advancements within the realm of ureterorenoscopy. A major advancement was made when flexible ureteroscopy came into being in the 1960s as it was first described by Marshall when a flexible fiber-optic pediatric cystoscope was used to access the upper urinary tract. ${ }^{1}$ Several limitations existed with this iteration though, which included the absence of a working channel and lack of active deflection to direct the scope. Through the 1980s, scope technology evolved with the ability to run irrigation fluid and an actively deflecting tip as described by Bagley et al. ${ }^{2}$ Today, modern flexible ureteroscopes typically contain a working channel, the ability to run irrigation, and bidirectional actively deflecting tips.

\section{Flexible fiber-optic ureteroscopes and their limitations}

Prior to the introduction of digital flexible ureteroscopes, flexible fiber-optic ureteroscopes helped to reshape the minimally invasive options for management of urolithiasis in the collecting system. However, fiber-optic ureteroscopes are limited by the durability of the fiber-optic bundles themselves. The fiber-optic fibers are easily broken when passed through the ureter and during times of extreme deflection, such as entry into the lower pole. Using new Olympus 7.5 French flexible ureteroscopes, Pietrow et al showed that with an average of 15.3 passes, 20 or more optical fibers were broken. ${ }^{3}$ While the damaged ureteroscopes were described as being still usable, the damage was noted as it did present difficulties with adequately visualizing targets.

As the additional fibers continue to break, visualization continues to deteriorate until repair is ultimately required. The ureteroscopes can also develop a leak, which leads to fogging and usually complete failure requiring repair. Broken fibers not only limit the ability to perform ureteroscopy but also result in costly repairs. Tosoian et al reported that over a 1-year period in which 190 cases were performed, ureteroscope repair was 
required 20 times. $^{4}$ The total cost of repairs was $\$ 115,000$ (an average of $\$ 5,750$ per repair or $\$ 605$ per case when spread out over the 190 cases). Furthermore, a repaired ureteroscope has been shown to have a shorter lifespan before the next repair is required relative to a new ureteroscope. Carey et al described new flexible fiber-optic ureteroscopes having a lifespan of $40-48$ uses before requiring repair. ${ }^{5}$ Following repair, these same ureteroscopes required repair after only 11.1 uses.

The visualization of small stones and tumors within the upper tract can be limited by the contrast of the fiber-optic image. Fiber-optics produce a honeycomb pattern due to the spaces between the fiber-optic strands. ${ }^{6}$ In an effort to improve these limitations of fiber-optic technology, digital ureteroscope development began to occur.

\section{Digital flexible ureteroscope technology}

It was through the use of digital image sensors that digital ureteroscopy was made possible, and there are two different imaging chips that have been used in the development of these scopes: charge-coupled devices (CCDs) and complementary metal oxide semiconductors (CMOS). Both imagers function by converting photons into electrons. ${ }^{7}$ There are wires within the ureteroscope that carry the digital signal to the image processor, which is then converted into the image for real time viewing. ${ }^{8}$ CMOS imagers require less energy, process images faster, run at cooler temperatures, and are less expensive to produce when compared with $\mathrm{CCD} .^{7}$ In contrast, CCD imagers are a mature technology that has high sensitivity and is less affected by signal noise. ${ }^{7}$ Digital imagers were continually miniaturized until small enough to be applied to ureteroscopy when the chips were placed on the tip of the ureteroscope with the industry coining the phrase "chip on the tip" and "chip on the stick". The image quality of digital ureteroscopes is so advanced that Humphreys et al described this as a "new world revealed" as they visualized Randall's plaques within the renal papilla. ${ }^{8}$ A comparison between fiber-optic images and video images is shown in Figure 1.

Because digital ureteroscopy utilizes the "chip on the tip" to collect information and relay it to the endoscopy tower, the bulky camera head required for nondigital endoscopic procedures is eliminated (Figure 2). In the author's experience, the digital ureteroscopes offer improved ergonomics by being lighter and, therefore, easier to handle, something that is especially important during longer procedures where hand fatigue can become an issue. Furthermore, the reduction in scope cords from two (camera and light cord) to one helps to prevent cord entanglement and reduces clutter in the operative field. The combined cord and the elimination

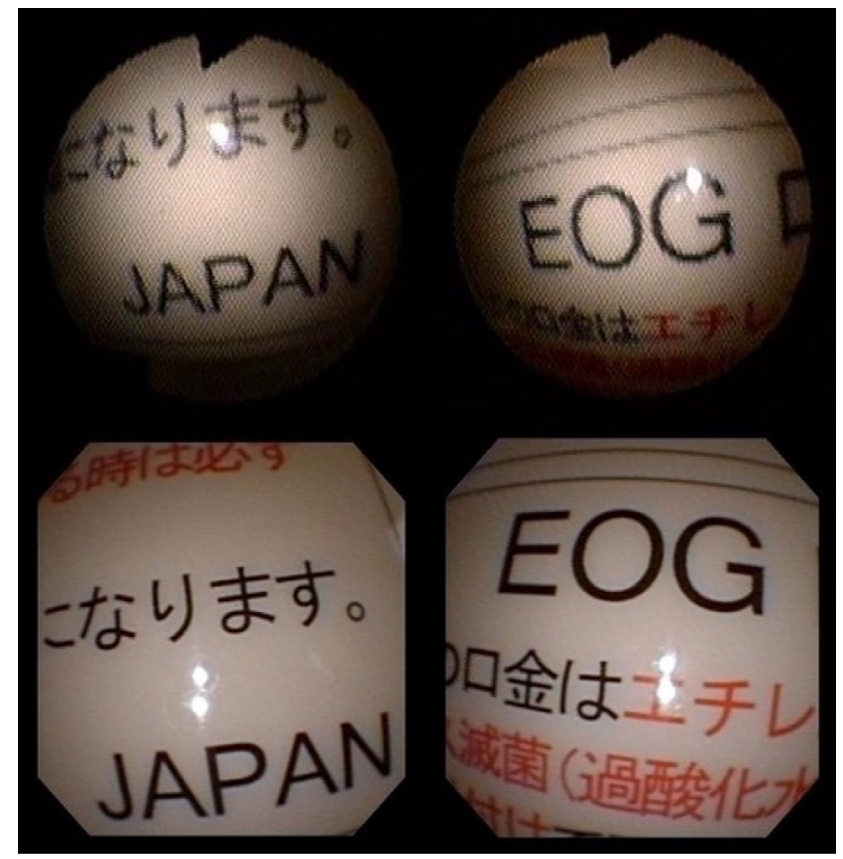

Figure I Images from Olympus URF-P5 (fiber-optic) on top, Olympus URF-V (digital) on bottom.

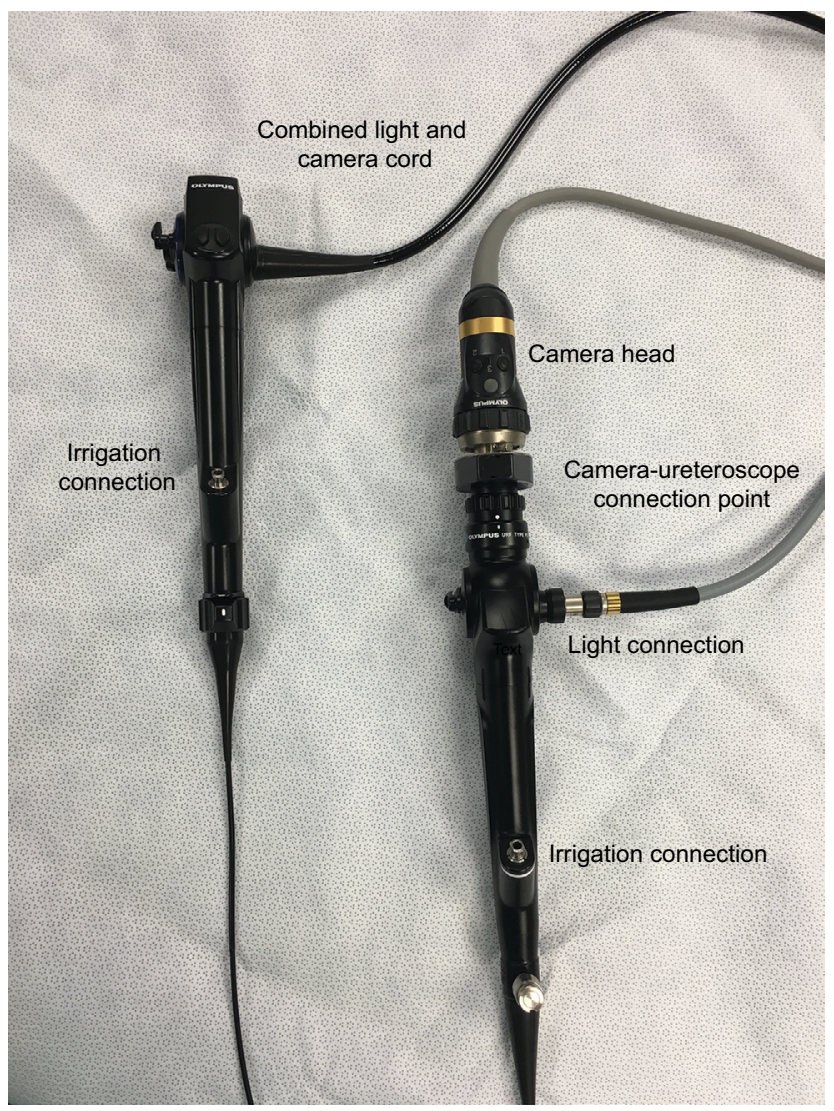

Figure 2 Olympus URF-V digital (left) and Olympus URF-P5 fiber-optic (right) flexible ureteroscope.

of the bulky camera head does result in a connection that is specific to the manufacturer. A digital ureteroscope then must be used with the respective manufacturer's tower. 
Most CMOS-based digital ureteroscopes utilize LED light sources at the tip of the digital ureteroscope, which eliminates the need for an external light source. LED bulb life is $\sim 10,000$ hours, which is $10-20$ times longer than Xenon light sources. ${ }^{9}$ Fiber-optic illuminating systems, such as Xenon light sources, can also pose an increased fire risk when the light cable is left exposed near a surgical drape. ${ }^{10-12}$ The CMOS-based digital flexible ureteroscopes do not require a detachable light cable because of the LED light sources that eliminate this risk of fires and patient burns. All components are integrated into one connector (Figure 3). While both the Olympus URF-V and URF-V2 continue to use an external light source, the light cable is integrated into the ureteroscope cable head decreasing the risk of fires.

\section{Subjective comparison of digital to fiber- optic flexible ureteroscopes}

Several studies have compared fiber-optic to digital ureteroscopes. A comparison was done between the fiber-optic flexible ureteroscope 11274AA (Karl Storz Endoscopy, Tuttlingen, Germany) and the digital flexible ureteroscope URF-V (Olympus Medical System, Tokyo, Japan) in 44 consecutive ureterorenoscopies (22 consecutive with the fiberoptic scope and 22 consecutive with the digital scope). ${ }^{13}$ Maneuverability and visibility were rated subjectively on a

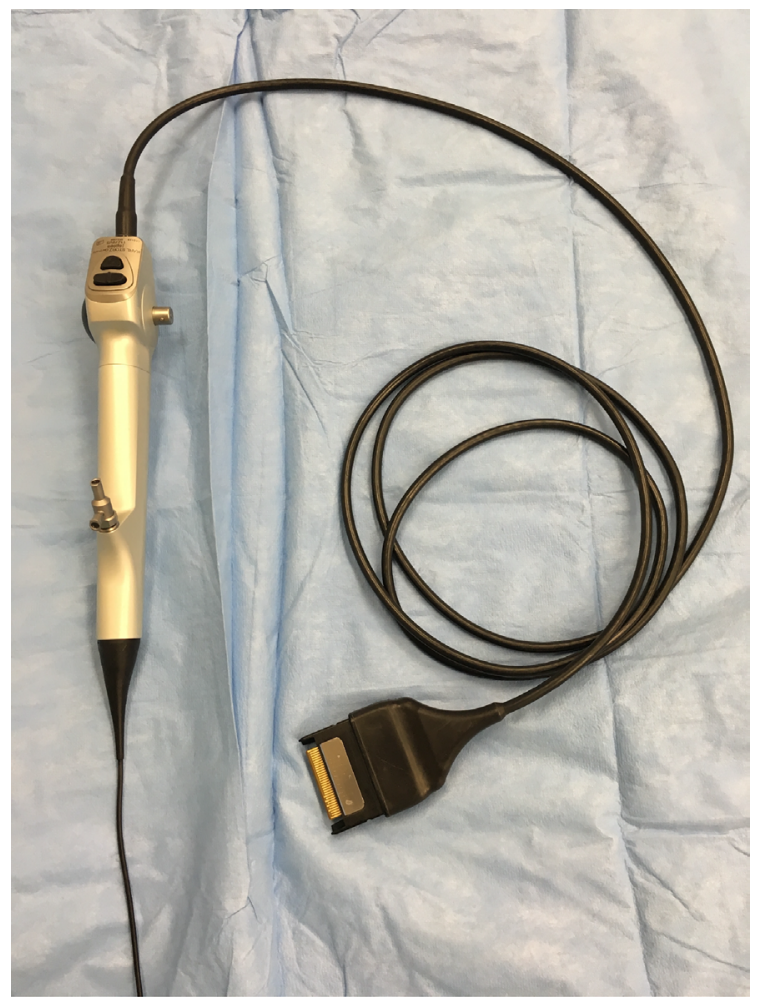

Figure 3 Karl Storz Flex Xc flexible digital ureteroscope. scale from 1 to 5 . The ability to access the lower pole of the kidney was also recorded. The digital scope rated higher for both maneuverability and visibility. It was also noted to have higher clarity, a lack of moiré effect, and superior magnification abilities. Furthermore, the digital ureteroscope was able to visualize the entire collecting system in $90.9 \%$ of cases versus $81.8 \%$ for the fiber-optic ureteroscope.

\section{Clinical performance of digital versus fiber-optic flexible ureteroscopes}

Several studies have compared the clinical performance of fiber-optic versus digital flexible ureteroscopes. Somani et al compared the URF-V (Olympus Medical System) to the URF-P5 (Olympus) during 118 ureteroscopic procedures for nephrolithiasis and found similar stone-free rates at 1 month postoperatively, which being $86 \%$ versus $88 \%$, respectively. ${ }^{14}$ The mean operative time was noted to be longer for the fiberoptic URF-P6 compared to the digital URF-V, at 53.8 versus 44.5 minutes, respectively. Binbay et al compared outcomes between a fiber-optic ureteroscope, the Flex-X2 (Karl Storz) and a digital ureteroscope, the DUR-D (ACMI Gyrus, Southborough, MA, USA) and reported similar findings. ${ }^{15}$ The fiber-optic ureteroscope and digital ureteroscope had similar stone clearance rates of $88.2 \%$ and $85.7 \%$, respectively, but the operative time was longer for the fiber-optic ureteroscope as compared to the digital ureteroscope at 46.5 and 38.3 minutes $(P=0.001)$, respectively.

\section{Limitations of digital flexible ureteroscopes}

There are some drawbacks of digital ureteroscopes. Early digital ureteroscopes had image distortion due to acoustic wave production during laser lithotripsy, but this was reduced by the implementation of shock absorbers at image sensor. ${ }^{16}$ In the authors' experience, image distortion can still occur with modern digital ureteroscopes due to the photoacoustic effect of the holmium:YAG laser when activated near the tip of the ureteroscope.

Digital ureteroscopes possess relatively larger distal tips compared to fiber-optic ureteroscopes. Most current generation digital ureteroscopes have a tip diameter of 7.7-8.7 French, which can be a limiting factor when attempting to access the upper tracts in patients. The BOA and COBRA System (Richard Wolf, Knittlingen, Germany) digital ureteroscopes currently have the smallest tip diameters of 6.6 and 5.2 French, respectively, but there has been no data published commenting on the use of these ureteroscopes in vivo. Several studies have reported the inability to pass 
a digital ureteroscope to the target when presented with a narrow infundibulum with subsequent successful passage of a flexible fiber-optic ureteroscope, indicating the benefit of maintaining a smaller fiber-optic flexible ureteroscope as a backup. ${ }^{7,8,13}$

Additionally, some urologists have described potential difficulty orienting a digital ureteroscope within the renal collecting system owing to a lack of the pendulum camera attached to the eyepiece. ${ }^{17}$ As a possible solution to this limitation, the Roboflex Avicenna (Elmed USA, Orlando, FL. USA) is a robot technology that incorporates a flexible ureteroscope and allows the surgeon to drive the ureteroscope from an ergonomic console. Saglam et $\mathrm{al}^{17}$ found that this technology was able to subjectively reduce operator strain. Future platforms will likely continue to explore the incorporation of robotics into endoscopic procedures.

\section{Failure rates and need for repair}

There have been several reports describing the durability of digital ureteroscopes. Early flexible ureteroscopes often suffered from poor durability. Afane et al showed an average life span of 6-15 cases prior to requiring servicing for fiber-optic flexible ureteroscopes smaller than 9 French. ${ }^{18}$ Through the use of certain techniques, such as the use of a ureteral access sheath, moving stones out of lower calyx prior to laser lithotripsy, and utilizing a 200 micron Holmium laser fiber, Pietrow et al were able to extend fiber-optic flexible ureteroscopes to an average of 27 uses. ${ }^{3}$ As the fibers of a fiber-optic ureteroscope are fragile, a digital ureteroscope by comparison may be more resilient due to the lack of the fiber-optic bundle but may also be subject to different patterns of failure. Major repairs are required of digital ureteroscopes when there is significant loss of deflection, when the light source fails, or when structural damage from extended use occurs. Al-Qahtani et al were able to perform 60 cases with an Olympus URF-V digital ureteroscope until repair was required by utilizing an access sheath and relocating lower pole stones in certain cases ${ }^{19}$ Using three new Karl Storz Flex-Xc digital flexible ureteroscopes, Multescu et al extended their first ureteroscope to 96 consecutive procedures by employing strategies, such as lower pole stone relocation; further, when the ureteral access sheath was removed with the deflection mechanism kept beyond of the end the ureteral access sheath, ureteroscope life was extended to greater than 150 uses in the two subsequent ureteroscopes. ${ }^{20}$

With regard to loss of deflection, Multescu et al reported that the Olympus URF-V digital ureteroscope experienced no loss of deflection after 22 cases while in comparison, the fiber-optic Storz 1127AA ureteroscope lost 10 degrees of flexion. ${ }^{13}$ Multescu et al also commented on the deflection loss during a comparison of the Flex-Xc (Karl Storz) digital flexible ureteroscope, the URF-V (Olympus Medical Systems), and the Cobra (Richard Wolf) fiber-optic flexible ureteroscope. Each ureteroscope was used during 30 different cases with the URF-V losing 5\% (14 degrees) of deflection while the Flex-Xc and the Cobra lost 9\% (24 degrees) and $10 \%$ (27 degrees), respectively. ${ }^{21}$

Furthermore, flexible ureteroscopes can be significantly damaged when a laser fiber is pulled back within the ureteroscope channel while the laser is activated. Xavier et al described a new technology whereby the CMOS imager of the DUR-D digital flexible ureteroscope (Gyrus ACMI, Southborough, MA) was able to detect the colored cladding of a laser fiber and communicate this information to an endoscopic protection system that would turn off the laser if the fiber was pulled back within the ureteroscope. ${ }^{22}$ This system worked $100 \%$ of the time in turning off the laser before ureteroscope damage, but did suffer a high false shutdown rate in 6/10 patients in which the system was tested.

Digital ureteroscopy is still a relatively new technology and ongoing technologic improvement is occurring. As the components of the scope are further miniaturized the overall scope size will be further reduced. Mass production should help lower costs and with improvements in design durability might be improved. It is likely that digital technology will completely replace fiber-optic technology for flexible ureteroscopes in the future.

\section{Cost of Digital Flexible Ureteroscopes}

Digital ureteroscopes are comparatively more expensive than fiber-optic versions. When Binbay et al compared the digital DUR-D Invisio (Gyrus ACMI) to the fiber-optic FlexX2 (Karl Storz), the cost differences were large: purchase cost $\$ 90,000$ versus $\$ 30,000$, respectively, and repair cost of $\$ 36,000$ versus $\$ 20,000$, respectively. ${ }^{15}$ At the time of their publication, Al-Qahtani et al purchased their Olympus URF-V for 60,000 Euro $(\sim 85,000)$ with a repair cost of 25,000 Euro $(\sim 35,000) .{ }^{19}$ As the technology matured and additional digital ureteroscope models were made available, the prices have been reduced. At the time of preparation of this manuscript, flexible digital ureteroscopes list pricing is $\sim \$ 25,000$ USD without a manufacturer contract.

As described earlier, flexible ureteroscopy can have significant costs associated with processing, maintenance, and repairs. Tosoian et al calculated costs of $\$ 605$ per use over a 1-year period in which 190 procedures were completed. ${ }^{4}$ 
Cases done in the outpatient setting were calculated as returning a net profit while cases done in the inpatient setting possessed a net loss. Cases were profitable until repair costs reached an average of $\$ 1,200$ per case. These procedures were all done using fiber-optic technology. Martin et al performed a comprehensive evaluation of the costs associated with digital ureteroscopy using the Karl Storz Flex Xc digital ureteroscope. ${ }^{23}$ When used over a period of 160 cases, a cost of $\$ 848$ per use was established, not including initial purchase cost. The break-even point between profit and loss was reached at 99 cases.

The financial viability of incorporating flexible digital ureteroscopy into a urologic practice is complex, but likely to be profitable when associated costs of the ureteroscope can be spread out over a large ureteroscopic case load. Ureteroscope purchase cost, repair costs, case volume, scope longevity, procedure length, sterilization, etc, should all be factored into the cost of using flexible ureteroscopes.

\section{Technical specifications}

There are currently multiple manufacturers that produce digital flexible ureteroscope platforms including Karl Storz, Olympus, Wolf, and most recently Boston Scientific (Marlborough, MA, USA). There are both similar and unique attributes of the ureteroscopes produced by these manufacturers.

Gyrus ACMI released the first digital ureteroscope in $2006,{ }^{24}$ the Invisio DUR-D digital flexible ureteroscope. Due to the limitations of chip technology at the time, the tip diameter of the ureteroscope was 8.7 French and the shaft diameter was 9.3 French, larger than comparable fiber-optic flexible ureteroscopes at the time. The relatively larger diameter could pose an issue when attempting to access calyces with narrow infundibula. The technology held promise though, and Gyrus ACMI was acquired by Olympus in $2008 .^{25}$

Olympus currently offers two different digital ureteroscopes, the URF-V and the URF-V2, but the URF-V is being phased out. Both are based on CCD chip technology. The URF-V2 is an improvement on the original URV-V as it has a smaller diameter shaft (8.4 versus 9.9 French, respectively) and improved deflection capabilities $\left(275^{\circ}\right.$ up and $275^{\circ}$ down versus $180^{\circ}$ up and $275^{\circ}$ down, respectively). Tip diameter is the same between both versions at 8.5 French. Additionally, Olympus is the only manufacturer that offers narrow band imaging (NBI), a technology that has been shown aid in the detection of urothelial tumors. NBI utilizes light in two specific wavelengths (415 and 540 $\mathrm{nm})$, which are absorbed by hemoglobin and bring attention to surface capillaries and submucosal blood vessels that can be associated with increased vascularity of tumors. Traxer et $\mathrm{al}^{26}$ demonstrated a $22.7 \%$ improvement in tumor detection rate when using NBI as $14 \%$ of diagnosed tumors were only visible with NBI.

Karl Storz produces a single model of a digital ureteroscope, the Flex-Xc. This ureteroscope is similar in size to the other available digital ureteroscopes with tip and shaft diameters of 7.9 and 8.5 French, respectively, and utilizes CMOS technology. It is built with a unique, ovoid distal tip shape while most other ureteroscopes typically have a circular construction. This ovoid shape is believed to provide improved drainage during ureteroscopy. ${ }^{16}$ Karl Storz also offers imaging technology aimed at improving visualization. The Storz Professional Image Enhancement System (SPIES) includes several modalities: Spectra A, Spectra B, Clara, and Chroma. ${ }^{27}$ Spectra A and Spectra B adjust the wavelength of light to improve contrast. Clara adjusts brightness to improve vision in dark areas of an image. Chroma adjusts the sharpness of an image. The effect of the SPIES is currently being studied in the clinical setting.

Richard Wolf has two offerings of CMOS-based digital ureteroscopes, the COBRA System and the BOA System. The main difference between these two ureteroscopes is the tip diameter of 5.2 and 6.6 French, respectively, and the shaft diameter of 9.9 and 8.7 French, respectively. The smaller tip diameter may facilitate passage of the ureteroscopes up the ureter and into the kidney. It may also improve access to calyces that possess narrow infundibula. The shaft diameters are similar to ureteroscopes from other manufacturers though, and clinical studies will need to be carried out to establish a benefit of the smaller diameter tip with regards to accessibility during ureterorenoscopy. An additional feature of the COBRA System is dual working channels. There is a 2.4-French laser fiber and irrigation channel in addition to the standard 3.6-French working and irrigation channel. This allows for simultaneous use of a basket and laser fiber while maintaining high levels of irrigation.

A new CMOS-based single-use flexible digital ureteroscope, the LithoVue ${ }^{\mathrm{TM}}$ (Boston Scientific) has recently become available and is positioned as an alternative to reusable ureteroscopes. This ureteroscope possesses a tip diameter of 7.7 French and a shaft diameter of 9.5 French. The LithoVue ureteroscope has been compared to both a fiberoptic (URF-P5) and digital (URF-V) flexible ureteroscope in a cadaveric model. The performance of the LithoVue was found to be satisfactory and similar in function to the reusable ureteroscopes. ${ }^{28}$ The introduction of 
Table I Digital ureteroscope comparison

\begin{tabular}{|c|c|c|c|c|c|c|c|}
\hline & Invisio DUR-D & Flex-Xc & URF-V & URF-V2 & LithoVue & BOA & COBRA \\
\hline Manufacturer & Gyrus ACMI & Karl Storz & Olympus & Olympus & Boston Scientific & Richard Wolf & Richard Wolf \\
\hline Availability & No & Yes & Yes & Yes & Yes & Yes & Yes \\
\hline Tip diameter (Fr) & 8.7 & 7.9 & 8.5 & 8.5 & 7.7 & 6.6 & 5.2 \\
\hline Shaft diameter (Fr) & 9.3 & 8.5 & 9.9 & 8.4 & 9.5 & 8.7 & 9.9 \\
\hline Deflection (up/down) & $250 / 250$ & $270 / 270$ & $180 / 275$ & $275 / 275$ & $270 / 270$ & $270 / 270$ & $270 / 270$ \\
\hline Working channel & 3.6 & 3.6 & 3.6 & 3.6 & 3.6 & 3.6 & $2.4 / 3.6$ \\
\hline Chip technology & CMOS & CMOS & CCD & CCD & CMOS & CMOS & CMOS \\
\hline Disposable & No & No & No & No & Yes & No & No \\
\hline $\begin{array}{l}\text { Approximate list price } \\
\text { (USD)** }\end{array}$ & $\mathrm{N} / \mathrm{A}$ & $\$ 25,700$ & Phasing Out & $\$ 24,990$ & $\$ 3,000$ & $\$ 23,800$ & $\$ 25,900$ \\
\hline
\end{tabular}

Note: **Significant discounts are available with manufacturer contracts.

Abbreviations: CCD, charge-coupled device; CMOS, complementary metal oxide semiconductors; Fr, French; N/A, not applicable.

a single-use flexible ureteroscope may dramatically alter how we think about flexible ureteroscopy and its associated costs. With a single-use scope, the surgeon will have maximal deflection and baseline optics at the start of every procedure. Given the disposable nature of the scope, perhaps more challenging cases will be undertaken since the concern for breaking the scope and paying the subsequent repair costs becomes a nonfactor. The human resources to process and handle the scope between procedures are also not needed, but resources to order the scopes and stock the shelves are needed. For institutions that have a limited number of reusable scopes, the single-use scope may allow for additional procedures to be performed in a day and reduce opportunity costs in the operating room waiting for a scope to be reprocessed. However, the question remains of whether the high cost of the single-use scope ( $\$ 1500-\$ 3000$ USD) will offset the benefits of eliminate the processing and repair costs. Table 1 shows a further breakdown of digital ureteroscope specifications.

\section{Conclusion}

Over the past decade, we have seen a migration from fiberoptic ureteroscopes to digital systems. As technology continues to improve, it is likely that at some point in the future all ureteroscopes will be digital. Over the next decade, the transition to higher resolution scopes such as 4 and $8 \mathrm{k}$ devices will likely occur. Perhaps more importantly, high dynamic range systems that allow for a wider color gamut could become available. This may help to address some of the limitations in visibility that occur at times with digital systems.

\section{Disclosure}

BEK is a consultant for Boston Scientific, Bard Surgical, and ThermDX; Course proctor for Coloplast Instructional Course. The authors report no further conflicts of interest in this work.

\section{References}

1. Marshall VF. Fiber optics in urology. J Urol. 1964;91:110-114.

2. Bagley DH, Huffman JL, Lyon ES. Flexible ureteropyeloscopy: diagnosis and treatment in the upper urinary tract. J Urol. 1987; 138(2):280-285.

3. Pietrow PK, Auge BK, Delvecchio FC, Silverstein AD, Weizer AZ, Albala DM, Preminger GM. Techniques to maximize flexible ureteroscope longevity. Urology. 2002;60(5):784-788.

4. Tosoian JJ, Ludwig W, Sopko N, Mullins JK, Matlaga BR. The effect of repair costs on the profitability of a ureteroscopy program. J Endourol. 2015;29(4):406-409.

5. Carey RI, Gomez CS, Maurici G, Lynne CM, Leveillee RJ, Bird VG. Frequency of ureteroscope damage seen at a tertiary care center. JUrol. 2006; 176(2):607-610.

6. Aslan P, Kuo RL, Hazel K, Babayan RK, Preminger GM. Advances in digital imaging during endoscopic surgery. J Endourol. 1999; 13(4):251-255.

7. Shah K, Monga M, Knudsen B. Prospective randomized trial comparing 2 flexible digital ureteroscopes: ACMI/Olympus Invisio DUR-D and Olympus URF-V. Urology. 2015;85(6):1267-1271.

8. Humphreys MR, Miller NL, Williams JC Jr, Evan AP, Munch LC, Lingeman JE. A new world revealed: early experience with digital ureteroscopy. J. Urol. 2008;179(3):970-975.

9. Andonian S, Okeke Z, Smith AD. Digital ureteroscopy: the next step. J Endourol. 2008;22(4):603-606.

10. Smith LP, Roy S. Fire/burn risk with electrosurgical devices and endoscopy fiberoptic cables. Am J Otolaryngol. 2008;29(3):171-176.

11. Eggen MA, Brock-Utne JG. Fiberoptic illumination systems can serve as a source of smoldering fires. J Clin Monit Comput. 1994; 10(4):244-246.

12. No author listed. OR fires caused by fiberoptic illumination systems. Hazard Health Devices 1982;11(7):148-149.

13. Multescu R, Geavlete B, Georgescu D, Geavlete P. Conventional fiberoptic flexible ureteroscope versus fourth generation digital flexible ureteroscope: a critical comparison. J Endourol. 2010;24(1): 17-21.

14. Somani BK, Al-Qahtani SM, de Medina SD, Traxer O. Outcomes of flexible ureterorenoscopy and laser fragmentation for renal stones: comparison between digital and conventional ureteroscope. Urology. 2013;82(5):1017-1019.

15. Binbay M, Yuruk E, Akman T, et al. Is there a difference in outcomes between digital and fiberoptic flexible ureterorenoscopy procedures? J Endourol. 2010;24(12):1929-1934.

16. Alexander B, Fishman AI, Grasso M. Ureteroscopy and laser lithotripsy: technologic advancements. World J Urol. 2015;33(2):247-256.

17. Saglam R, Muslumanoglu AY, Tokatli Z, et al. A new robot for flexible ureteroscopy: development and early clinical results (IDEAL stage 1-2b). Eur Urol. 2014;66(6):1092-1100. 
18. Afane JS, Olweny EO, Bercowsky E, et al. Flexible ureteroscopes: a single center evaluation of the durability and function of the new endoscopes smaller than 9Fr. J Urol. 2000;164(4):1164-1168.

19. Al-Qahtani SM, Geavlette BP, Gil-Diez de Medina S, Traxer OP. The new Olympus digital flexible ureteroscope (URF-V): initial experience. Urol Ann. 2011;3(3):133-137.

20. Multescu R, Geavlete B, Georgescu D, Geavlete P. Improved durability of flex-Xc digital flexible ureteroscope: how long can you expect it to last? Urology. 2014;84(1):32-35.

21. Multescu R, Geavlete B, Geavlete P. A new era: performance and limitations of the latest models of flexible ureteroscopes. Urology. 2013; 82(6):1236-1239.

22. Xavier K, Hruby GW, Kelly CR, Landman J, Gupta M. Clinical evaluation of efficacy of novel optically activated digital endoscope protection system against laser energy damage. Urology. 2009;73(1): $37-40$.

23. Martin CJ, McAdams SB, Abdul-Muhsin H, et al. The economic implications of a reusable flexible digital ureteroscope: a cost-benefit analysis. J Urol. Epub 2016 Sep 27.
24. "Gyrus ACMI launches the World's First Distal Sensor Flexible Ureteroscope.” Business Wire. Available from: http://www.businesswire.com/ news/home/20060918005977/en/Gyrus-ACMI-launches-WorldsDistal-Sensor-Flexible. Accessed October 20, 2016.

25. "Milestones." Olympus America. Available from: http://www.olympusamerica.com/corporate/corp_milestones.asp. Accessed October 20, 2016.

26. Traxer O, Geavlete B, Gil-Diez de Medina SG, Sibony M, Al-Qahtani SM. Narrow-band imaging digital flexible ureteroscopy in detection of upper urinary tract transitional-cell carcinoma: initial experience. J Endourol. 2011;25(1):19-23.

27. Kamphuis GM, de Bruin DM, Fallert J, Gultekin MH, de Reijke TM, MP LP. Storz professional image enhancement system: a new technique to improve endoscopic bladder imaging. J Cancer Sci Ther. 2016;8:071-077.

28. Proietti S, Dragos L, Molina W, Doizi S, Giusti G, Traxer O. Comparison of new single-use digital flexible ureteroscope versus nondisposable fiber optic and digital ureteroscope in a cadaveric model. J Endourol. 2016;30(6):655-659.
Research and Reports in Urology

\section{Publish your work in this journal}

Research and Reports in Urology is an international, peer-reviewed, open access journal publishing original research, reports, editorials, reviews and commentaries on all aspects of adult and pediatric urology in the clinic and laboratory including the following topics: Pathology, pathophysiology of urological disease; Investigation and treatment of

\section{Dovepress}

urological disease; Pharmacology of drugs used for the treatment of urological disease. The manuscript management system is completely online and includes a very quick and fair peer-review system, which is all easy to use. Visit http://www.dovepress.com/testimonials.php to read real quotes from published authors. 